\title{
Desarrollo científico en Colombia: una revisión de las revistas científicas especializadas en ciencias de la naturaleza. 1950-1999
}

\section{Scientific development in Colombia: a revision about scientific journals specialized in natural science. 1950-1999}

\author{
Franco Moreno, R. A ${ }^{1}$., Gallego Badillo, $\mathbf{R}^{2}$. y Pérez Miranda, $\mathbf{R}^{2}$.
}

${ }^{1}$ Licenciado en Química de la Universidad Pedagógica Nacional. Bogotá, D. C. Colombia. ${ }^{2}$ Profesores de la Universidad Pedagógica Nacional. Bogotá, D. C. Colombia. Grupo de Investigación Representaciones y Conceptos Cientúficos - IREC- frankoupn@gmail.com, grupo irec@yahoo.es

\section{Resumen}

El presente artíaulo se deriva de la realización de un proyecto de investigación, dentro de la línea de "Historia social de las ciencias y de la educación en ciencias". Con base en la metodología suministrada por la cienciometría y bibliometría, se examinaron las publicaciones de científicos colombianos en las revistas internacionales "Journal of the American Chemical Society", en el "American Joumal of Physics", en "The Joumal of Experimental Biology", y en las nacionales "Revista Colombiana de Química", "Revista Colombiana de Física" y "Actualidades Biológicas". Hechos los análisis documentales comparativos, la conclusión es que durante el período señalado, no se puede afirmar que en el país se haya organizado una comunidad cient́fica.

\section{Palabras Clave}

Historia social de las ciencias, epistemología, comunidad cient́fica, cienciometría, bibliometría.

\section{Abstract}

This paper was bom from the development of a research project related to the line of "social history and education in sciences". It is based on the methodology given by the scienciometry and bibliometry, taking into account publications of Colombian scientists on international journals such as "Joumal of the American Chemical Society", "American Joumal of Physics", "The Joumal of Experimental Biology", and national as "Revista Colombiana de Química", "Revista Colombiana de Física" and "Actualidades Biológicas". After doing the documental comparative analysis, the condusion is that during the pointed term, it is not possible to affirm that this country has organized a scientifically community.

\section{Keywords}

Social science History, epistemology, scientifically community, scienciometry, bibliometry.

\section{Introducción}

La historia social de las ciencias, también denominada historia extema en oposición a la historia interna del desarrollo del conocimiento cient́fico (Laudan, 2005; Shapin, 2005), suministra argumentos para explicitar las caractenísticas del desarrollo de la actividad científica en un país determinado. La historia social tiene que ver con los estudios de los factores sociales, culturales, políticos y económicos que inciden significativamente en la institucionalización de las ciencias y de la actividad cientúfica, entre los cuales cabe mencionar la creación de programas académicos para la formación de las generaciones de científicos de relevo, la redacción y publicación de textos de enseñanza (Bowler y Morus, 2007; Gallego Torres, Gallego Badillo y Pérez Miranda, 2009). 
La historia intema a su vez, está referida al análisis de la lógica de las estructuras conceptuales y metodológicas de cada uno de los modelos cient́íicos que fueron sustituidos, de la de los que los sustituyeron y de las razones también lógicas que condujeron a esa sustitución. Dentro de esta perspectiva se adoptaría la propuesta popperiana (Popper, 1962), la Khuniana (Kuhn, 1972) o la Lakotasiana (Lakatos, 1983).

La publicación en 1962 del libro de T. S. Kuhn que se cita, inició la conciliación entre los representantes de la historia interna y los de la historia interna. Además, en oposición a la aproximación positivista de una ciencia desarrollada por individuos geniales (Gallego Torres, 2002), aislados e independientes del proceso de formación escolar (Toulmin, 1977), Kuhn introdujo la categoría epistemológica explicativa de comunidad científica, para una aproximación de mayor credibilidad de las historias tanto internas como externas del conodimiento científico.

Los estudios históricos que se adelantaron después del artículo de I. Lakatos (1983) de 1972, llevaron a conduir que no había una historia de las ciencias, sino reconstrucciones de cada hecho científico asumido como objeto de investigación. A partir de esta puntualización, distintos especialistas en cada una de las ciencias de la naturaleza, se ocuparon de reconstruir la historia de sus ciencias particularmente y bajo el convencimiento de una pluralidad histórica (Estany, 2005), aquella concepción de un modelo de ciencias, entre los especialistas se acabó.

Todos estos trabajos condujeron finalmente a la condusión de que la pregunta por qué era la ciencia en general, fuera un interrogante que no debía de ser formulado, ya que solo podía ser absuelto desde las particularidades de cada una de las ciencias de la naturaleza. Ciencia pasó a ser aquello que se publica en las revistas especializadas y científico aquel personaje, aquel miembro de un colectivo a quien se le admite un artículo en una de esas revistas y que, además, es referenciado por otros colectivos en revistas diferentes, del mismo tenor (Barona, 1994). Desde la historia social las ciencias fueron asimiladas como sistemas de producción de conocimiento (Bemal, 1976). Estos fueron los fundamentos conceptuales que dirigieron la presente investigación.

Desde la década de 1980 , se han suscitado preguntas acerca del desarrollo de la actividad científica a nivel Latinoamericano en general, y de Colombia en particular, desde perspectivas del pensamiento humano (Puerta, 1989). Es desde esta época que se ha intensificado la necesidad de hacer estudios que midan de una manera sistemática la producción cientíica en el país, reconociendo en las publicadiones existentes en las revistas especializadas un importante indicador de la productividad cient́fica (Melo, 1986). Una indagación de este corte, por una parte, se inscribe en los estudios sociales de la ciencia, y se sustenta desde los supuestos metodológicos de la cienciometría (Barona, 1994), enfoque metodológico que se apoya en el citerio de la bibliometría (Callon, Courtial y Penan, 1995); a la vez que propicia la discusión alrededor de la pertenencia como indicador de la productividad científica en las sociedades (Gallego Badillo, 2008).

\section{Referentes Teóricos}

Historia y Epistemología de las ciencias

La investigación en Historia y Epistemología de las ciencias tiene un lugar importante dentro de la Nueva Didáctica de las Ciencias, y las investigaciones que en este campo se realizan, tienen su fundamento epistemológico dentro de las elaboraciones que al respecto han tenido lugar durante el siglo XX hasta la actualidad, construcciones que convergen en la búsqueda de explicaciones sobre la naturaleza de la ciencia, desde puntos de vista que se contraponen a concepciones empiropositivistas (Popper, 1962; Bachelard, 1979; Lakatos, 1983; Kuhn, 1972 y Gieré, 1998) 


\section{Las Comunidades Científicas}

La categoría de comunidad científica es introducida por T.S Kuhn en 1972 como altemativa al auestionado paradigma objetivista de la lógica positivista, reconciliando las elaboraciones que desde los puntos de vista internalistas y externalistas de la ciencia han estado separados. La base de una comunidad cientúfica la constituyen los grupos de investigación de Universidades, Centros de Investigación, de Empresas, y de otras entidades que compiten y convierten a la ciencia en mercancía. Así, se acepta que las comunidades cientúficas están configuradas por académicos que por su producción de conocimiento pertenecen a estas comunidades.

En relación con la comunidad cient́fica actual, Bowler y Rhys (2005) señalan que, esta comienza a configurarse en las primeras décadas del siglo $X X$, bajo la potencial influencia de la segunda guerra mundial y la guerra fría. Desde esta perspectiva, en la comunidad científica actual, se acepta que el imaginario tradicional del cient́fico como personaje aislado de sus comunidades está cambiando, tomando fuerza la idea de la actividad científica como fenómeno social, cultural y político.

La Actividad Científica en Colombia

Con relación a la constitución de comunidades científicas a nivel Latinoamericano en General, y de Colombia en particular, hay acuerdos sobre el hecho de que, la actividad científica se ha desarrollado sin la existencia de un pensamiento cientúfico crítico, independiente, "modemo", a cambio de el autoengaño y la autonegación que el caudillismo ha establecido bajo el rótulo de la independencia. En la misma dirección, es de señalar que hemos sido simples consumidores, primero de productos, y luego, de tecnologías y ciencia, lo que hace pensar que la ciencia siempre ha llegado a Colombia convertida en mercancía.

Por su parte, Melo. J, (1986), desde una perspectiva escéptica, pero realista, afirma que el despegue de la actividad científica en el país se lleva a cabo entre la década del 60 y ochenta, poniendo de manifiesto algunos problemas que han obstaculizado el desarrollo cient́fico ligado a la investigación.

\section{Revistas Científicas Especializadas}

Según la Organización de las Naciones Unidas para la Educación, la Ciencia y la Cultura, UNESCO, una revista se caracteriza por ser "una publicación periódica que presenta especialmente artíaulos científicos, escritos por autores diferentes, e información de actualidad sobre investigación y desarrollo de cualquier área de la ciencia"(Lozano, 1996: 3).

Por su parte, A. Schaffner (1995) señala que las revistas científicas son publicaciones periódicas que aparecen a mediados del siglo XVII en el marco de la revolución industrial, en particular, gracias al perfeccionamiento de la imprenta, inventada desde el siglo XV. En este sentido, las revistas científicas cumplen roles importantes como: la construcción de una base colectiva de conocimiento, la comunicación de la información, la validación de la calidad de la investigación, la distribución de retribuciones o premios, y la construcción de comunidades científicas, entre otras. Pero la verdadera función de estas revistas cobra mayor importancia en el sentido de la creación del conocimiento impreso,

\section{Referentes Metodológicos}

Este trabajo de revisión documental, se inscribe en el campo de investigación sobre historia y epistemología de las ciencias (Gallego Badilllo, $R$ y Pérez Miranda, R, 1996), vinculando los estudios sociales de la ciencia, y adoptando la cienciometría como opción metodológica. 


\section{Cienciometría y Bibliometría}

La cienciometría, o "ciencia de la ciencia", se entiende como el estudio de la documentación científica, política y sociológica de la ciencia, que con todos los cuestionamientos que ha recibido, hoy constituye uno de los recursos para historiar la ciencia desde el punto de vista social, al punto que existen acuerdos sobre la idea de que: ciencia es lo que está publicado en una revista científica especializada, y cient́fico es quien allí tiene una publicación (Barona, 1994; Macías, 2000). Con respecto a la bibliometría, existen acuerdos que permiten interpretarla como una herramienta que desarrolla modelos y mediciones matemáticas para el estudio cuantitativo de la producción cientúfica-investigativa que circula en las revistas cient́ficas, (Callon, Courtial \& Penan, 1995; Barona, 1994).

\section{Diseño Metodológico}

Selección de las revistas científicas especializadas

Para esta revisión documental se hizo una consulta preliminar de las bases de datos que contienen información espeáfica sobre las revistas cient́ficas especializadas. Estas bases de datos agrupan las revistas indexadas con mayores factores de impacto a nivel mundial, como se presenta a continuación.

\begin{tabular}{|c|c|}
\hline Base de Datos & Cantidad de Revistas de Química, Física y Biología, \\
\hline Science Citation Index & $\mathbf{1 5 1}$ \\
\hline Latindex & $\mathbf{4 4}$ \\
\hline
\end{tabular}

Tabla No. 1. Cantidad de revistas científicas en SCI y Latindex.

Una vez consultada la disponibilidad de las revistas enlistadas en las bases de datos: Science Citation Index y Latindex, se procedió a consultar la existencia de estas revistas en los catálogos de la biblioteca de la Universidad Nacional de Colombia, de la biblioteca del Banco de la RepúblicaLuis Ángel Arango y de la Universidad de los Andes. De la base de datos Saience Citation Index se seleccionó una revista representativa por cada ciencia como se expresa en la tabla No. 2.

\begin{tabular}{|c|c|c|c|}
\hline \multirow{2}{*}{$\begin{array}{l}\text { Base de Datos } \\
\text { Mundial }\end{array}$} & \multicolumn{3}{|c|}{ Títulos Seleccionados } \\
\hline & Qúlmica & Física & Biología \\
\hline $\begin{array}{l}\text { Science Citation } \\
\text { Index }\end{array}$ & $\begin{array}{l}\text { Journal of the } \\
\text { American Chemical } \\
\text { Society }\end{array}$ & $\begin{array}{c}\text { American } \\
\text { Journal of Physics }\end{array}$ & $\begin{array}{l}\text { Journal of Experimental } \\
\text { Biology }\end{array}$ \\
\hline
\end{tabular}

Tabla No 2 Revistas Seleccionadas a partir del SCI

Con respecto a las revistas Colombianas, se seleccionó una revista representativa por cada ciencia, en la base de datos Latindex, información que se muestra en la tabla No. 3.

\begin{tabular}{|c|c|c|c|}
\hline \multirow{2}{*}{$\begin{array}{l}\text { Base de Datos } \\
\text { Iberoamericana }\end{array}$} & \multicolumn{3}{|c|}{ Títulos Seleccionados } \\
\hline & Química & Física & Biología \\
\hline Latindex & $\begin{array}{l}\text { Revista Colombiana } \\
\text { de Química }\end{array}$ & $\begin{array}{l}\text { Revista Colombiana de } \\
\text { Física }\end{array}$ & $\begin{array}{l}\text { Actualidades } \\
\text { Biológicas }\end{array}$ \\
\hline
\end{tabular}

Tabla No. 3. Revistas cient́ficas Colombianas Seleccionadas a partir de Latindex.

Recolección y Tratamiento de la información

Se han elaborado los instrumentos necesarios para la recolección de los registros, de conformidad con la intención de este trabajo. Por una parte, se construyeron las fichas bibliométricas para las revistas internacionales y nacionales (Anexo), a la vez que se adoptó el Resumen Analítico en Educación- RAE, como instrumento eficaz para la argumentación del análisis 
en la revisión documental. Se diseñó un instrumento que consiste en una matriz de categorías de análisis en cienciometría, siendo adoptadas las seis primeras desde uno de los referentes cienciométricos (Araújo y Arencibia, 2002). La información recopilada hizo necesario el complemento de las anteriores categorías, introduciendo los ítem 7 y 8 en la matriz. Como elementos de análisis dentro de las categorías se ha vinculado la bibliometría, determinada por los indicadores bibliométricos (Maćas, 2000) y los RAE, como se presenta a continuación:

\begin{tabular}{|c|c|c|c|}
\hline \multicolumn{2}{|c|}{ HISTORIA Y EPISTEMOLOGÍA DE LAS CIENCIAS } & Categorías de análisis & $\begin{array}{c}\text { Elementos de análisis: Indicadores } \\
\text { bibliométricos / RAES }\end{array}$ \\
\hline ESTUDIOS SOCIALES DE LAS CIENCIAS & AS CIENCIAS & $\begin{array}{l}\text { 1. El crecimiento cuantitativo de la } \\
\text { ciencia }\end{array}$ & $\begin{array}{l}\text { Cantidad de artículos de colombianos en las } \\
\text { revistas internacionales/ RAES }\end{array}$ \\
\hline \multicolumn{2}{|c|}{ CIENCIOMETRÍA } & & $\begin{array}{l}\text { Indicador de Edición y Colaboración; } \\
\text { Indicador de Región; Indicador de citas } \\
\text { bibliográficas / RAES }\end{array}$ \\
\hline BIBLIOMETRÍA & \multirow{2}{*}{$\begin{array}{l}\text { CATEGORÍAS } \\
\text { DE } \\
\text { ANÁLISIS }\end{array}$} & 3. La relación entre ciencia y tecnología & RAES \\
\hline & & 4. La estructura de comunicación entre & Indicador de Edición y Colaboración / RAES \\
\hline BASES DE DATOS & 4 & 5. Las relaciones entre el desarrollo & RAES \\
\hline & & científico y el crecimiento económico & \\
\hline Revistas Mundiales & $\downarrow$ & 6. La ciencia como actividad & Indicador de Metodología / RAES \\
\hline & LECTURA & $\begin{array}{l}\text { Investigativa: la productividad y } \\
\text { creatividad de los investigadores }\end{array}$ & \\
\hline Revistas Iberoamérica & CRITICA & 7. La producción científica como actividad & Indicador de Tipo de Autoría; Indicador de \\
\hline Revistas Colombia & & $\begin{array}{l}\text { Colectiva-comunitaria } \\
\text { desarrollo Cientifico }\end{array}$ & Indicador de Institución/ RAES \\
\hline
\end{tabular}

Cuadro No. 1. Diseño Metodológico de la Investigación. Matriz de Categorías.

\section{Resultados e Interpretación}

El siguiente es el panorama que se expone a partir del anterior cuadro de matriz de categorías y su correspondiente apoyo en los indicadores bibliométricos.

\section{El Crecimiento Cuantitativo de la Ciencia}

Como se muestra en las gráficas № 1 y 2, lo que se encuentra es que las publicaciones de colombianos en las revistas: Joumal of the American Chemical Society, American Joumal of Physics, American Joumal of Human Biology, no supera el $0.5 \%$ de la producción científica que allí circula. Por otra parte, no es sino hasta a mediados de la década de 1950 que aparecen artículos procedentes de Colombia en estas revistas, distinto de las publicaciones procedentes de países como España, México, Brasil y Argentina, cuya aparición comienza desde antes de la primera mitad del siglo XX, además, la presencia de artículos de estos países en su conjunto redondea el $3 \%$ de la participación, que si bien no es un indicio alentador, permite comenzar a establecer juicios acerca del desarrollo científico colombiano.

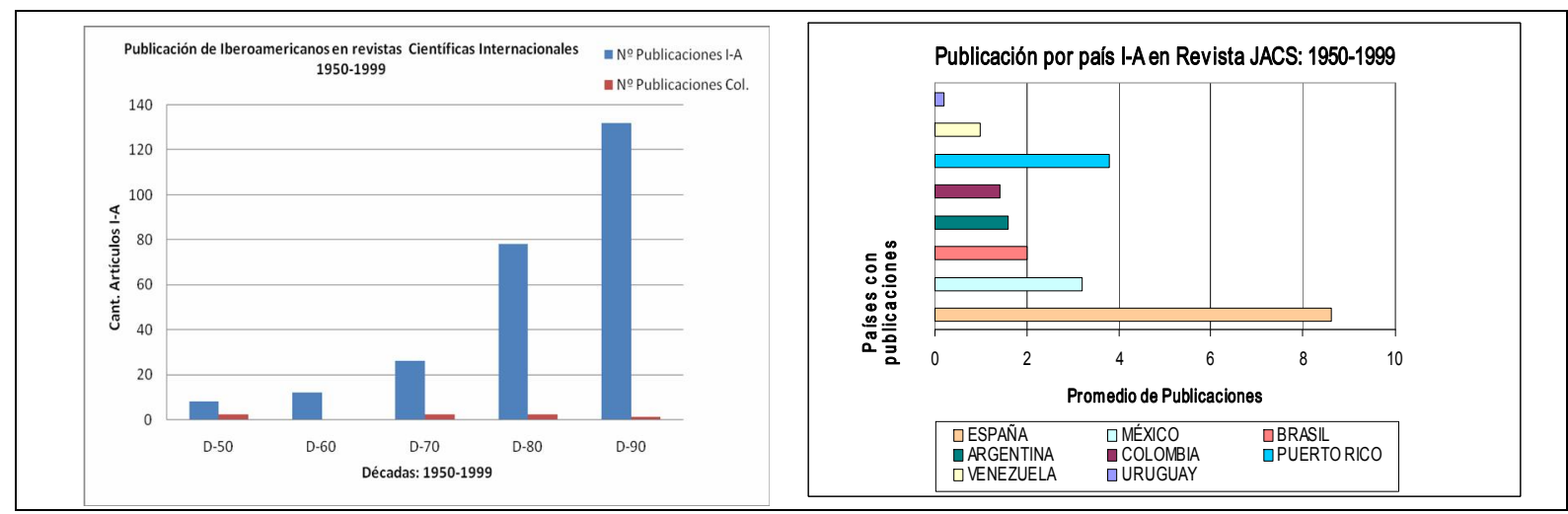

Gráficas No. 1 y 2 Panorama de la presencia Iberoamericana en las revistas cientúficas internacionales. 
Es de señalar que desde mediados de la década del ochenta se presenta un aumento generalizado de los artículos de Colombia en y Física y Química. Además, la mayoría de los artículos allí publicados hacen parte de las secciones dedicadas a notas de discusión y reflexiones frente a teorías, modelos y situaciones que en su momento eran objeto de estudio, y que aún hoy lo son. En este sentido, los aportes colombianos al desarrollo científico son poco significativos.

A pesar del aumento en la cantidad de artículos por edición durante la década de 1990, como lo muestra el indicador de edición y colaboración (gráfica № 3), la cantidad de artículos en colaboración no aumentó, manteniéndose la tendencia de las anteriores décadas, pero disminuyendo la presencia de extranjeros en la revista, lo cual es contradictorio con las intenciones estatales de esa época, que se centraban en el logro de la apertura hacia los mercados internacionales. De haber sido así, lo esperado sería un incremento en la cantidad de artículos en colaboración con cient́ficos de instituciones extranjeras. Más bien lo que refleja el indicador de edición y colaboración es el aumento en la necesidad de controlar procesos industriales en general, implementando los modelos y las teorías Químicas de conformidad con las exigencias que a nivel de productividad y competitividad exigía el aparato gubernamental de turno.

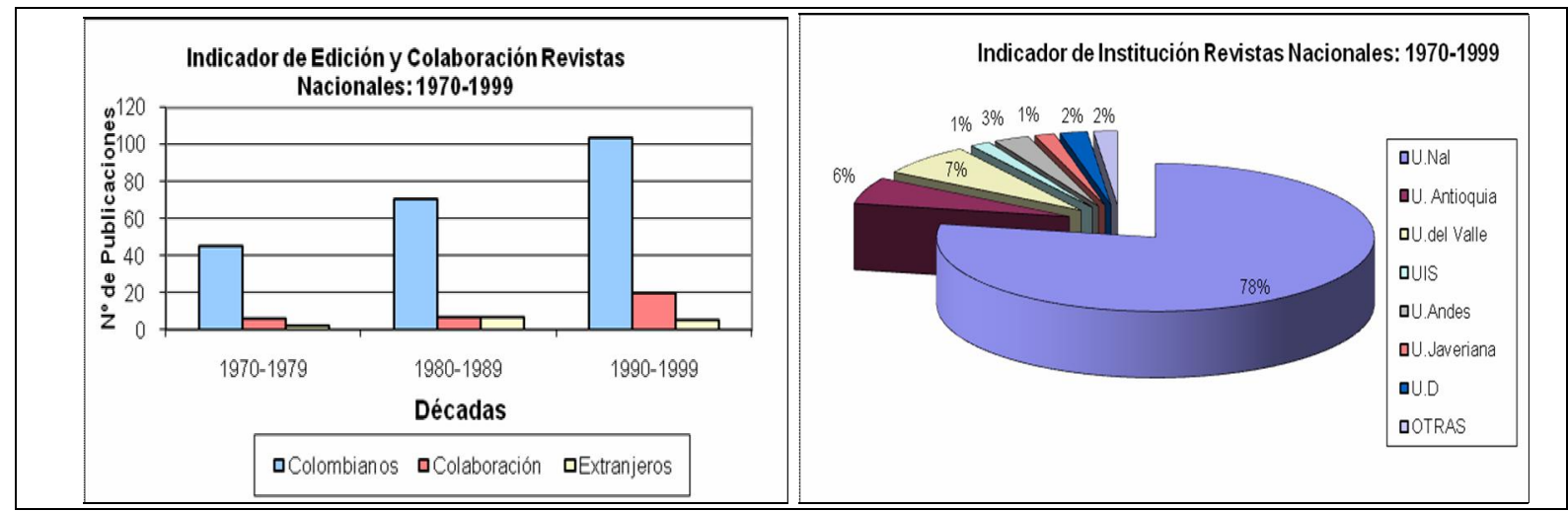

Gráficas No. 3 y 4. Indicadores de Edición-Colaboración e Institución en revistas nacionales: 1970-1999. Respectivamente.

La distribución geográfica de la producción académica se acentúa en las regiones de Cundinamarca, el Valle del Cauca, Antioquia y Santander respectivamente. Esto indica que la actividad cientúfica ha trascurrido en este orden de regiones en el país, pues geográficamente es en esos lugares es en donde están ubicadas tanto las universidades de mayor incidencia desde el punto de vista científico y tecnológico, como muchas de las industrias de gran influencia en la economía colombiana.

Como se presenta en el indicador de citas bibliográficas (gráfica № 5), es preciso reconocer un progresivo aumento en el número de citas de revistas cient́ficas, pues en la década de 1970 dominaba la tendencia en la cantidad de citas procedentes de libros de texto, comportamiento que cambia notablemente, siendo las revistas especializadas la mayor fuente de citas en la década de 1990. Así, se reconoce un crecimiento cuantitativo de la Química, puesto que la premisa para el ejercicio cienciométrico supone que, si la ciencia es lo que circula en las revistas especializadas, el correspondiente aumento o disminución del número de citas determinará dicho crecimiento (Barona, 1994). 


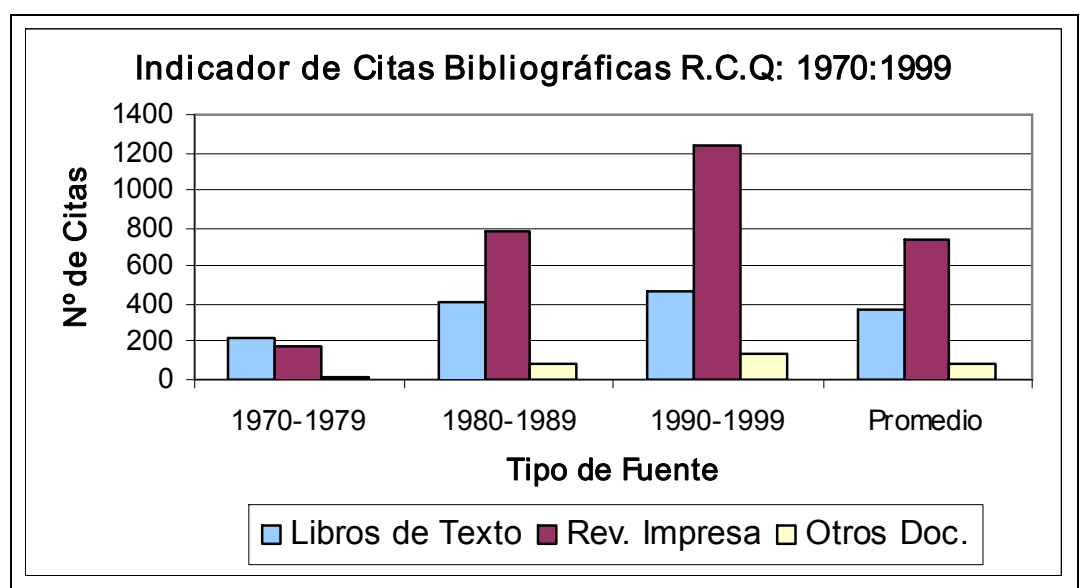

Gráfica No. 5. Indicador de citas bibliográficas R.C.Q

El Desarrollo de las Disciplinas y Subdisciplinas

La información bibliométrica recopilada muestra que en Química los trabajos se centraron en el análisis y la síntesis orgánica, siguiendo la síntesis bioquímica y la fitoquímica. En física las publicaciones versan sobre Mecánica Cuántica, Electromagnetismo, Termodinámica y Óptica, siendo la Física Nudear y la Relatividad las áreas de menor publicación. Las áreas de mayor movimiento en Biología han sido la Botánica y la Ecología, lo que hace pesar que su impulso obedece por una parte a la obra de Mutis, y por otra, a la trascendencia que en las dos últimas décadas han adquirido los asuntos relacionados con la conservación.

\section{La Relación entre Ciencia y Tecnología}

Bastaría con mencionar algunos ejemplos para mencionar que la mediana articulación entre la tecnología y la actividad cient́fica ha sido consecuencia del desarrollo económico del país, y el equipamento para la actividad experimental ha sido, por no decir que en su totalidad, traído del exterior, lo que hacía más sencillo y productivo el trabajo práctico, a la vez que se veían disminuidos los trabajos orientados a la construcción de equipos y dispositivos tecnológicos.

La Estructura de Comunicación entre los Científicos

El surgimiento de entidades que agrupan a los científicos entomo de un quehacer: la ciencia, es un factor de suma importancia en la consolidadción de comunidades científicas, hecho que en Colombia comenzó a darse desde la década de 1950 con la fundación de las sociedades de Química y Física. Por otra parte, es preciso afirmar que el hecho de publicar artículos científicos en conjunto con otras instituciones del orden local, nacional o internacional, es decir en colaboración, es un indicio tanto de la movilidad del discurso, como de la conformación de lazos académicos, y en últimas, de comunidades científicas. En este sentido, con todos los esfuerzos que desde la década de 1950 se hacían para consolidar esas relaciones, lo que se encuentra en Colombia desde la década de 1970 hasta 1999, es una comunicación muy discreta con los químicos del exterior, que se expresa en la cantidad moderada de artículos publicados en colaboración en la Revista Colombiana de Química. Sin embargo se reconoce la presencia de científicos extranjeros, la mayoría de ellos de Estados Unidos y Europa, hecho que evidencia el interés de estas naciones por la inversión y multiplicación del capital económico y humano en países en vía de desarrollo.

Las Relaciones entre el Desarrollo Científico y el Crecimiento Económico

La industria, por no decir que el mayor, es si uno de los más fuertes e influyentes sectores de la economía del país, y para su crecimiento ha sido necesario el desarrollo del conocimiento cient́fico, pero en mayor medida el tecnológico. En ese sentido, ha sido en ciertas regiones del país en donde se ha concentrado la actividad industrial: Cundinamarca, el Valle del Cauca, Antioquia y Santander lo han sido en potencia. Así, ésta es una razón de ser importante para pensar que es especialmente en estas regiones en donde se ha puesto a circular la mayor cantidad de 
conocimientos cient́ficos y tecnológicos necesarios para el funcionamiento de esa industria, lo cual guardaría relación con las universidades allí existentes, a la vez que se corresponde con los resultados bibliométricos en Química, Física y Biología, que indican que es de dichas regiones, respectivamente, de donde provienen los artículos científicos publicados en las Revistas especializadas.

La ciencia como actividad Investigativa: la productividad y creatividad de los investigadores

Tanto el análisis de la literatura científica que circula en las revistas científicas, como el indicador de metodología general que exhiben los artíaulos, son elementos de análisis de la producción científica como actividad investigativa; así, a continuación se presentan los resultados en cuanto a una clasificación general de la metodología:
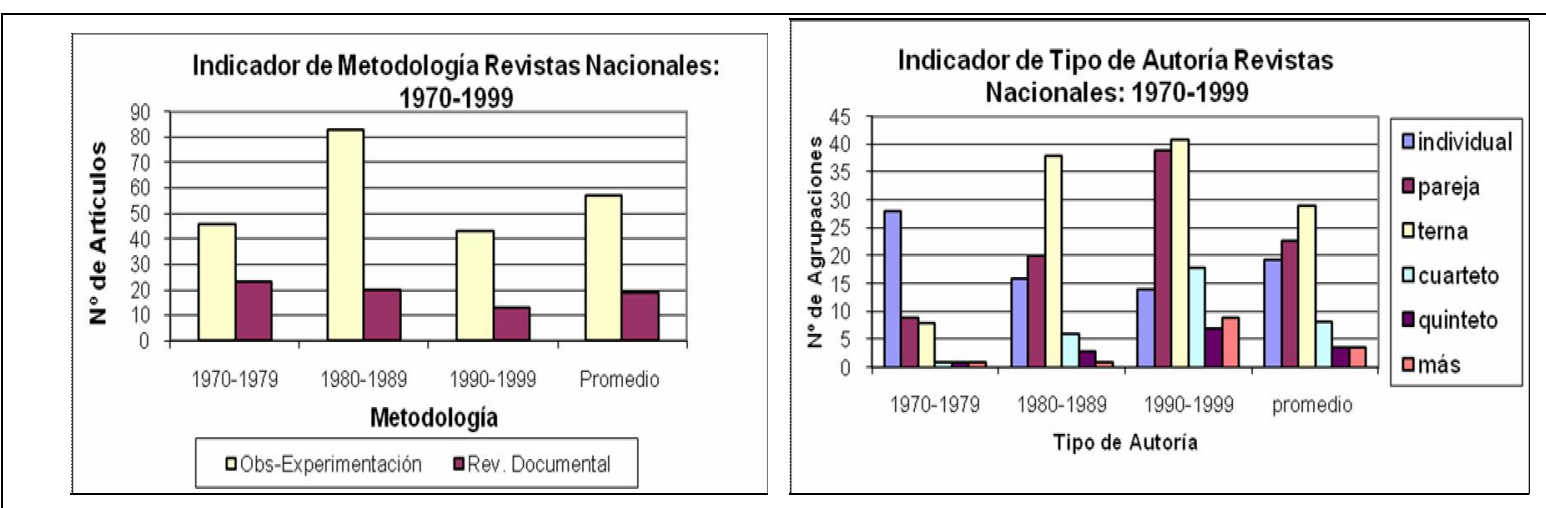

Gráficas № 6 y 7. Indicadores de Metodología y Tipo de autoría en revistas nacionales: 1970-1999. Respectivamente.

Como se muestra en la gráfica № 6 la gran mayoría de los trabajos publicados en ciencias de la naturaleza tiene una orientación metodológica centrada en la observación y la experimentación con una fuerte tendencia al desarrollo del trabajo práctico en el laboratorio, frente a pocos trabajos de revisión documental. En la mayoría de estos trabajos es evidente la preocupación por el rigor de los procedimientos en el laboratorio, a la vez que se refleja el seguimiento de los pasos del método cient́fico, lo aual se corresponde con una concepción empiropositivista de la ciencia y de la investigación, lo que limita la producción de conocimientos científicos centrados en lo hipotéticodeductivo (Gallego Badillo y Pérez Miranda, 1996).

\section{La Producción Científica Como Actividad Colectiva-Comunitaria}

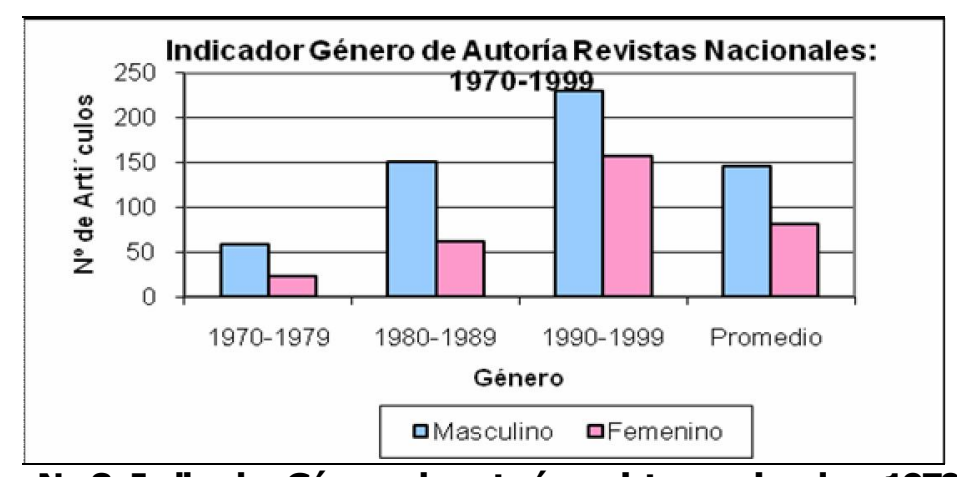

Gráfica No 8. Indicador Género de autoría revistas nacionales: 1970-1999

Entre los ciiterios más importantes para la apreciación de las comunidades científicas está la actividad colectiva, y lo que se encuentra en los indicadores de tipo de autoría en Química, Física y $\underline{T E \Delta}$ 
Biología, como se muestra en la gráfica № 7, es que entre las décadas de 1960 y 1970 predominan las publicaciones individuales, siendo muy pocas las agrupaciones en parejas y ternas, y mucho menores o nulas en algunos casos, las publicaciones por grupos de cinco o más autores. Ya en la década de 1980 se muestra una tendencia al aumento de publicaciones por parejas y temas siendo aun escasas las agrupaciones en cuartetos o grupos de más personas. Sin embargo en Física y Biología predominan los trabajos individuales sobre los colectivos. En la década de 1990 predominan los trabajos por parejas y temas en Química y Biología, no obstante, en Física la tendencia de publicación individual no fue superada por las agrupaciones de autores.

Este comportamiento a nivel de tipo de autoría como indicador de la actividad científica colombiana en colectivos de trabajo, denota cambios en el imaginario del cient́fico sobre la ciencia. Así, se asume aquí, que desde la década de 1970 predomina el individualismo y el aislamiento de los hombres de sus comunidades, hecho que tendrá algún viraje en la década de 1980 cuando los cient́ficos se agrupan en pequeños colectivos de trabajo que aumentarán según lo ya anotado. Esto indica que comenzaba a consolidarse una comunidad de especialistas alrededor de la actividad cientúfica con un objeto de estudio en común, lo aual seguramente obedecerá a lo que internacionalmente venía ocurriendo con las comunidades cient́ficas. No obstante, la actividad científica en el país centrada en la investigación sigue en tela de juicio. Por otra parte, lo que muestra el indicador de género de autoría (Gráfica № 8) es la exclusión histórica de la mujer en los procesos de construcción científica, hecho que ha tendido a transformarse en las últimas dos décadas, sin perjuicio de que sean los hombres quienes siguen dominando tales tendencias.

Incidencia de las Instituciones en el desarrollo Científico

Como ya se anotó, los artículos producidos por colombianos y publicados en las revistas Journal of the American Chemical Society, American Joumal of Physics y The Joumal of Experimental Biology, son de autores pertenecientes a las Universidades Nacional de Colombia, de Antioquia, del Valle, de los Andes e Industrial de Santander, lo cual las sitúa como centros primordiales de la actividad cientúfica del país. De hecho las sociedades y otras entidades de gran importancia en cuanto a la agrupación de especialistas en Química, guardan toda o alguna relación con estas instituciones.

Lo anterior cobra mayor validez al contrastarlo con el indicador bibliométrico de institución, el aul muestra que es en la Universidad Nacional de Colombia en donde tienen lugar la mayor cantidad de contribuciones que circulan en las Revistas de Física y Química, seguida de las Universidades del Valle, de Antioquia, Industrial de Santander (UIS) y de los Andes respectivamente. Las demás instituciones que registran alguna contribución hacen parte del ítem otras. Como ya se discutió en el indicador de región, la Universidad de Antioquia muestra la mayor agrupación de publicaciones en Biología.

Desde mediados de la década de 1960 el desarrollo científico colombiano ha sido liderado por Colciencias, razón por la cual esta institución tiene un gran significado en el desarrollo de la mayoría de los proyectos de investigación en ciencias de la naturaleza, y aryos productos circulan en las revistas científicas. Por otra parte, el ICFES aparece como la entidad que apoya algunos proyectos de investigación, mediante convenios suscritos con entidades del exterior como el Banco Interamericano de Desarrollo (BID) -Programa ICFES-BID y la Organización de Estados Americanos (OEA). El Ministerio de Educación Nacional es mencionado en los agradecimientos de algunos trabajos. Como entidad local en la Universidad Nacional, el Centro de Investigaciones y Desarrollo Cient́fico, CINDEC se reporta en varias publicaciones.

Además, en Química, en su momento, sobresale el apoyo de instituciones como el Laboratorio Químico Nacional, destacándose también entidades como el Instituto Colombiano Agropecuario (ICA), y el Instituto Geográfico Agustín Codazzi en la cofinanciación de algunos proyectos de investigación. 
El Instituto de Ciencias Naturales ICN con su Museo de Historia Natural, es la entidad que ha liderado los trabajos de investigación en Biología, seguido de unidades académicas como el Instituto, de Investigaciones Marinas y Costeras, INVEMAR y el Instituto Alexander von Humboldt. En Física, es influyente el Observatorio Astronómico fundado en 1946, y el Instituto de Asuntos Nucleares ayyo funcionamiento no superó la década de 1980.

Es de señalar que son muy pocos, por no decir que ningunos, los registros en los cuales se agradece o se menciona el apoyo de la empresa privada nacional en el desarrollo de trabajos e investigaciones cient́ficas. Por el contrario, el apoyo de instituciones intemacionales como universidades, laboratorios y algunas industrias sí se ve reflejado en la literatura.

\section{Conclusiones}

Adelantada la revisión de las revistas especializadas en ciencias de la naturaleza, tres internacionales y tres nacionales, y procesada la información de conformidad con los criterios cienciométricos, se encuentra que, la presencia de colombianos en las revistas científicas internacionales especializadas en Química y Física es mínima en comparación con toda la producción internacional, a la vez que a nivel Iberoamericano están por encima de Colombia cuatro países. Más desalentador es el panorama en Biología, pues en la revista internacional revisada no hay artículos de colombianos, lo cual permite establecer que la pertenencia colombiana a la comunidad cientŕfica internacional no se ha inidado.

En el marco nacional, los especialistas colombianos constituyen la gran mayoría de las publicaciones frente a una baja circulación de trabajos en colaboración y aún menor es la presencia de contribuciones extranjeras, lo aual reafirma la idea de la no pertenencia de Colombia a la comunidad científica intemacional. Con respecto a la existencia de revistas científicas en el país, hay que anotar que son muy pocas las existentes como medios de circulación del discurso científico nacional, en comparación con países de la región como México, Brasil y Argentina, lo que se traduce en un desarrollo cientúfico pequeño.

La cienciometría constituye una importante opción conceptual y metodológica para hacer historia de la ciencia. Así, la investigación documental reportada permite establecer que durante la segunda mitad del siglo XX en Colombia la actividad cientúfica en Química, Física y Biología se ha llevado a cabo obedeciendo, en esencia, a las necesidades industriales, agrícolas y tecnocientíficas, lo cual posibilitó la formación de profesionales en estas áreas en distintos niveles de especialidad.

La actividad científica en Colombia ha tenido lugar gracias al aprendizaje y la transferencia del desarrollo científico y tecnológico ocurrido en los países avanzados. Dicha transferencia se ha logrado por una parte, gracias a la incursión de una muy reducida minoría de profesionales en los sistemas educativos de los países desarrollados mediante programas académicos de internacionalización y algunos convenios de cooperación, a lo que se suma la inversión extranjera en la industria nacional y la correspondiente adopción de tecnologías y metodologías para la actividad científica también extranjeras.

La poco significativa presencia de colombianos en las revistas intemacionales especializadas en ciencias de la naturaleza, y la calidad de las publicaciones que circulan en las revistas nacionales da para pensar que si bien muchos de los especialistas en cada una de las ciencias de la naturaleza están agrupados en sociedades y en otras agremiaciones legalmente constituidas, estas en sí son unidisciplinares y al parecer sus finalidades se centran más en la reivindicación de las profesiones y la justificación del quehacer científico, lo que pone en entredicho la consolidación de la comunidad científica colombiana alrededor de la actividad investigativa, a la vez que se pone aún más en tela 
de juicio el proceder estatal y privado en cuanto al desarrollo científico y la consolidación de la comunidad cientŕfica del país.

Con estas apreciaciones no se asegura la ausencia de comunidad científica, de hecho haría falta explorar otros campos desde la sociología de la ciencia; además, la existencia de las agrupaciones mencionadas efectivamente evidencia la conformación de la comunidad, lo que sigue en discusión es ¿De qué comunidad se habla? ¿Qué especialistas la integran? ¿Desarrollan investigación y de Qué investigación se habla? Una respuesta desde el trabajo realizado sugiere que una comunidad cientŕfica ayyo eje sea la investigación no ha existido en el país.

\section{Bibliografía}

Barona, J. L. (1994). Ciencia e historia. Debates y tendencias en la historiografía.

Callon, M, Courtial, JP \& Penan, H. (1995). Cienciometría. El estudio cuantitativo de la actividad científica: de la bibliometría a la vigilancia tecnológica TREA. Gijón.

Gallego Badillo, R. (2008). Ciencias Revistas Especializadas y Comunidad Científica, Tecné Episteme y Didaxis, No. Extra, Universidad Pedagógica Nacional. 9-24.

Kuhn, T. S. (1972). La estructura de las revoluciones científicas. México: Fondo de Cultura Económica.

Lakatos, I (1983). La metodología de los programas de investigación científica. Madrid: Alianza.

Macías, C. (2002). Papel de la Informetría y de la Cienciometría y su Perspectiva Nacional e Internacional.

Puerta, J. (1989). El Pensamiento Científico en América Latina. Universidad de Antioquia, 58 (218). 15-20.

Anexo: Ficha bibliométrica para revistas científicas especializadas.

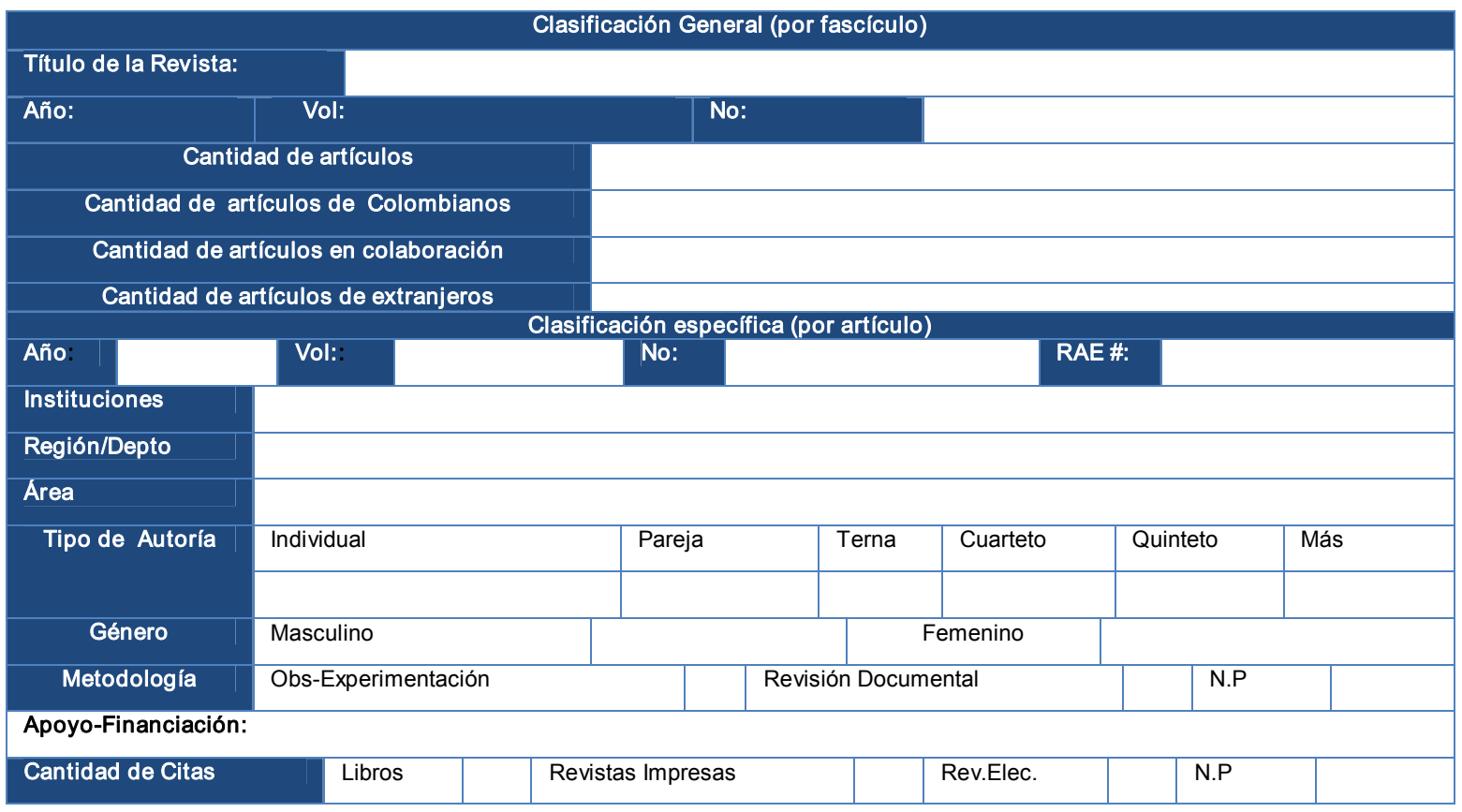

